\title{
Single incision laparoscopic surgery cholecystectomy in children - preliminary experience
}

\author{
Marcin Łosin, Piotr Czauderna, Andrzej Gołębiewski \\ Department of Surgery and Urology for Children and Adolescents, Medical University of Gdansk, Poland
}

Videosurgery and other miniinvasive techniques 2010; 5 (4): 139-141

DOI: 10.5114/wiitm.2010.18825

\begin{abstract}
Introduction: Laparoscopic cholecystectomy has become the gold standard for cholecystectomy, since Mouret introduced it in 1987. In 1997 Navarra described "one incision" cholecystectomy, but only recently has single incision laparoscopic surgery (SILS) gained wider acceptance, mostly due to technological developments. The primary goals of SILS are avoidance of visible scarring and minimizing surgical trauma.

Aim: We present our first experience of 3 cases of children (2 females, 1 male; ages 2.5-17 years) treated with SILS cholecystectomy.

Methods and results: One child had undergone previous open left adrenalectomy for neuroblastoma. We used a Covidien SILS Port in one case and 3 single-use low-profile ports in the others. Percutaneous stay suture was used to suspend the gallbladder, and standard cholecystectomy was performed using 1 straight and 1 disposable articulating instrument. There were no postoperative complications. Average operating time was $70 \mathrm{~min}$. Hospital stay varied from 2 to 4 days.

Conclusion: Our early experience with SILS cholecystectomy in children suggests that it is safe and effective, but further studies and greater numbers will be required to investigate the potential benefits of this approach.
\end{abstract}

Key words: cholecystectomy, laparoscopy, SILS, paediatric surgery

\section{Introduction}

Laparoscopy was one of the most significant surgical advances of the twentieth century and laparoscopic cholecystectomy has been recognized since 1987, when Phillipe Mouret performed the first videolaparoscopic cholecystectomy, as the gold standard procedure for gallbladder surgery [1]. During the last 20 years we have observed a tendency to minimize surgical trauma that challenged many surgeons to look for new approaches in laparoscopic surgery [2, 3]. Single incision laparoscopic surgery (SILS), in which all instruments are placed through a single, small umbilical incision, might be a step toward even less invasive surgical procedures. One of the major goals of introducing laparoscopic surgery was to reduce postoperative pain and improve cosmesis, so SILS may be considered as the next step to virtually "scarless" surgery $[4,5]$. We present 3 cases of paediatric patients treated in our department with the SILS procedure due to cholelithiasis.

\section{Material and methods}

In June 2009 SILS cholecystectomy and modified SILS cholecystectomy were performed in 3 children. There were 2 females and 1 male, and their age varied from 2.5 to 17 years. All patients presented with cholelithiasis confirmed by abdominal ultrasonography. The "single incision" approach was selected. We 
used a Covidien SILS Port in 1 case and 3 disposable low-profile ports in the others.

In the first case of an 8-year-old girl with Down syndrome a $2-\mathrm{cm}$ long longitudinal incision was made in the umbilicus and the abdominal cavity was opened, then the Covidien SILS Port was introduced. The abdominal cavity was inflated with carbon dioxide up to a pressure of $12 \mathrm{mmHg}$. After abdominal inspection, 2.0 Prolene percutaneous stay suture, on a straight needle, was placed through the gallbladder fundus to retract it. Using a special articulating instrument, Calot's triangle was exposed. After identification of all structures, both the cystic dust and cystic artery were clipped with 5-mm Haemolock clips. The gallbladder was resected in a retrograde fashion with a monopolar hook and removed in an "Endo-catch" sac through the umbilical incision. Immediately after surgery fluoroscopy revealed the presence of a small pneumothorax, probably due to relatively high stay suture placement, because of abnormal chest configuration. Before the patient's transfer from the operating room it was treated with single thoracentesis. The remaining hospitalization was uneventful.

The second child was operated on via 3 single-use low-profile ports introduced in the umbilicus. And in the last case, we used 2 disposable low-profile ports placed in the umbilicus. This was a child who had undergone previous open left adrenalectomy for neuroblastoma, so due to the presence of adhesions in the abdomen we decided to use an additional 3-mm instrument introduced percutaneously in the right epigastrium, instead of using 3 ports in the umbilicus. Suture was used to suspend the gallbladder, and standard cholecystectomy was performed using 1 straight and 1 disposable articulated instrument in both cases. There were no postoperative complications. Average operating time was 70 min. Hospital stay varied from 2 to 4 days.

\section{Discussion and conclusions}

Evolution of new techniques, such as robotic surgery, shows the demand for new approaches in surgery. One of the very visible trends is to minimize surgical trauma. Procedures such as NOTES (natural orifice transluminal endoscopic surgery) are controversial, particularly in children, but there is an obvious need among patients for "scarless" surgery [6-8]. Since NOTES requires perforation of a visceral organ, which is practically unacceptable in children, the SILS approach is gaining more popularity and may be a solution. Among papers dealing with SILS cholecystectomy in adults [9] there have been some reports of successful use of SILS procedures in the paediatric population, including SILS cholecystectomy [10, 11]. In our opinion SILS cholecystectomy should be considered more as a variation of LC than a new surgical technique, so there is no need to differentiate which procedure is better. Since SILS is a single-incision procedure, better cosmesis is obvious, especially when it is performed within the umbilicus. But there might be some limitations of this approach. Although in experienced hands SILS seems to be safe and feasible, the need for use of special articulated instruments, lack of instrument triangulation and operating mostly with the left hand make SILS more demanding. On the other hand there is always a possibility to convert to standard laparoscopy. The need to place percutaneous stay suture may lead to some complications, as observed in our series. Our early experience suggests that outcomes are probably comparable to standard laparoscopic cholecystectomy, but bigger patient series and potentially a prospective trial are necessary to assess the true benefits of this approach, as well as to investigate any potential advantages of this method over standard laparoscopic techniques.

\section{References}

1. Litynski GS. Profiles in laparoscopy: Mouret, Dubois, and Perissat: the laparoscopic breakthrough in Europe (1987-1988). JSLS 1999; 3: 163-7.

2. Navarra G, Pozza E, Occhinorelli S, et al. One wound laparoscopic cholecystectomy. Br J Surg 1997; 84: 695.

3. Piskun G, Rajpal S. Transumbilical laparoscopic cholecystectomy utilizes no incisions outside the umbilicus. J Laparoendosc Adv Surg Tech A 1999; 9: 361-4.

4. Cuesta MA, Berends F, Veenhof AA. The "invisible cholecystectomy": a transumbilical laparoscopic operation without a scar. Surg Endosc 2008; 22: 1211-3.

5. Tacchino R, Greco F, Matera D. Single-incision laparoscopic cholecystectomy: surgery without a visible scar. Surg Endosc 2009; 23: 896-9.

6. Chamberlain RS, Sakpal SV. A comprehensive review of singleincision laparoscopic surgery (SILS) and natural orifice transluminal endoscopic surgery (NOTES) techniques for cholecystectomy. J Gastrointest Surg 2009; 13: 1733-40.

7. Flora ED, Wilson TG, Martin IJ, et al. A review of natural orifice translumenal endoscopic surgery (NOTES) for intra-abdominal surgery: experimental models, techniques, and applicability to the clinical setting. Ann Surg 2008; 247: 583-602. 
8. Michalik M, Frask A, Trybull A, et al. Methods of treatment for gallbladder diseases. Videosurgery and other miniinvasive techniques 2009; 4: 121-5.

9. Kurpiewski W, Pesta W, Kowalczyk M, et al. SILS cholecystectomy - our first experiences. Videosurgery and other miniinvasive techniques 2009; 4: 91-4.

10. Ponsky TA, Diluciano J, Chwals W, et al. Early experience with single-port laparoscopic surgery in children. J Laparoendosc Adv Surg Tech A 2009; 19: 551-3.

11. Dutta S. Early experience with single incision laparoscopic surgery: eliminating the scar from abdominal operations. J Pediatr Surg 2009; 44: 1741-5. 